Revista Brasileira de Agricultura Irrigada v.12, no.7, p. 3090 - 3095, 2018

ISSN 1982-7679 (On-line)

Fortaleza, CE, INOVAGRI - http://www.inovagri.org.br

DOI: $10.7127 /$ rbai.v12n700969

Protocolo 969.18 - 14/08/2018 Aprovado em 28/02/2019

\title{
UTILIZAÇÃO DE DIFERENTES DISTRIBUIÇÕES DE PROBABILIDADE PARA PRECIPITAÇÕES MÀXIMAS NO MUNÍCIPIO DE FRANCISCO BELTRÃO/PR
}

\author{
Allan Remor Lopes $^{1 *}$, Camila Moreno Giarola ${ }^{2}$, Marcelo Dotto ${ }^{3}$, Acir Felipe Grolli Carvalho ${ }^{4}$, \\ Alvaro Rodrigo Freddo ${ }^{5}$, Elouize Xavier ${ }^{6}$
}

\begin{abstract}
RESUMO
O conhecimento das precipitações máximas diárias anuais e de seu tempo de retorno em uma região são de interesse prático para a gestão de recursos hídricos. Nesse contexto, o presente trabalho teve como objetivo avaliar a distribuição das precipitações diárias máximas anuais para o município de Francisco Beltrão, no Paraná. Foram utilizados dados de precipitação de 1974 a 2017 (44 anos), sendo determinadas as precipitações máximas pela distribuição de Gumbel e Log Pearson III para os períodos de retorno de 2, 5, 10, 20, 50 e 100 anos. Para os menores períodos de retorno (2, 5 e 10 anos), a Distribuição Log Pearson III, apresentou maiores índices de precipitação máxima, enquanto que para os períodos de retorno de 25, 50 e 100 anos a Distribuição de Gumbel apresentou maiores índices de precipitação máxima. Ambas as distribuições de probabilidade apresentaram modelos de regressão similares e satisfatórios, no entanto a distribuição de Gumbel obteve um melhor ajuste dos dados $\left(\mathrm{r}^{2}=\right.$ $92,7 \%)$.
\end{abstract}

Palavras-chave: tempo de retorno, gestão dos recursos hídricos, hidrologia.

\section{USE OF DIFFERENT PROBABILITY DISTRIBUTIONS FOR MAXIMUM PRECIPITATION IN THE MUNICIPATILITY OF FRANCISCO BELTRÃO/PR}

\footnotetext{
ABSTRACT

The knowledge of the annual maximum daily precipitation and its time of return in a region are of practical interest for the management of water resources. In this context, the present work had as objective to evaluate the distribution of annual maximum daily precipitation for the municipality of

${ }^{1}$ Doutorando em Engenharia Agrícola, Professor, União de Ensino do Sudoeste do Paraná. Dois Vizinhos-PR. E-mail: allanremorlopes@gmail.com *Autor para correspondência

${ }^{2}$ Acadêmica, Universidade Paranaense. Umuarama-PR. E-mail: camila_moreno_giarola@hotmail.com

3 Doutor em Agronomia, Professor, União de Ensino do Sudoeste do Paraná. Dois Vizinhos-PR. E-mail: marcelodotto@unisep.edu.br

4 Doutor em Agronomia, Professor, União de Ensino do Sudoeste do Paraná. Dois Vizinhos-PR. E-mail: acirfgcarvalho@gmail.com

5 Doutor em Agronomia, Professor, União de Ensino do Sudoeste do Paraná. Dois Vizinhos-PR. E-mail: alvaro@unisep.edu.br

6 Doutora em Agronomia, Professora, União de Ensino do Sudoeste do Paraná. Dois Vizinhos-PR. E-mail: elouize@unisep.edu.br
} 
Francisco Beltrão, Paraná. Precipitation data from 1974 to 2017 (44 years) were used, and maximum precipitation was determined by the Gumbel and Log Pearson III distributions for the return periods of 2, 5, 10, 20, 50 and 100 years. For the smaller return periods (2, 5 and 10 years), the Log Pearson III Distribution presented higher rates of maximum precipitation, while for the 25,50 and 100 years return periods, Gumbel Distribution presented higher maximum precipitation. Both probability distributions presented satisfactory and similar regression models. However, the Gumbel distribution obtained a better adjustment of the data $(\mathrm{r} 2=92.7 \%)$.

Keywords: return period, water resources management, hydrology.

\section{INTRODUÇÃO}

A precipitação pode ser definida como toda água que provém do meio atmosférico e atinge a superfície terrestre (MIRANDA et al., 2017). No sistema agrícola, representa o principal meio da entrada de água, sendo fundamental para o entendimento da dinâmica hídrica do meio e pode ser utilizada como um indicador de épocas mais adequadas para a semeadura (BARRETO et al., 2014).

Para Carvalho et al. (2013), caracterizar a distribuição da pluviosidade é de extrema importância para a agricultura, pois a demanda hídrica de uma planta varia de acordo com a espécie, estágio de desenvolvimento e demanda atmosférica.

A precipitação máxima é definida como a ocorrência extrema com duração, distribuição temporal e espacial crítica para uma área e pode atuar na erosão do solo, inundações em áreas urbanas e rurais, obras hidráulicas, entre outros (BERTONI; TUCCI, 2015). Sendo assim, o estudo de eventos de precipitações máximas tem grande relevância para a gestão de recursos hídricos (BESKOW et al., 2015).

No dimensionamento de obras hidráulicas e drenagem agrícola, deve-se conhecer as precipitações máximas esperadas para aquela região, de forma que a estrutura planejada resista adequadamente suportando tal valor extremo de precipitação (SANTOS et al., 2014). Sendo a chuva uma variável aleatória contínua, pode-se representa-la através de alguma distribuição teórica de probabilidade (ALVES et al., 2013).

Para Moruzzi e Oliveira (2009) não existe um melhor método para determinação de precipitações máximas pelo tempo de retorno, porém a distribuição de Gumbel é a mais aceita. Para Cotta et al. (2016), a distribuição de Gumbel é amplamente utilizada em diversas áreas do conhecimento para modelar os valores máximos de ocorrência de um determinado fenômeno.

Para Back (2001), muitos autores utilizam a distribuição de Gumbel, assumindo a hipótese que os dados amostrais seguem a distribuição de Gumbel, sem procurar outra hipótese que poderia proporcionar um melhor ajuste. No entanto, existem diversas distribuições de probabilidade, e Valverde et al. (2004) enfatizam que a distribuição Log Pearson III está entre as distribuições de Probabilidade mais indicadas e utilizadas para hidrologia.

Devido a grande carência de informações relativas a precipitações máximas para o município de Francisco Beltrão no Paraná, este trabalho teve o objetivo de determinar as precipitações máximas para diferentes períodos de retorno através da distribuição de Gumbel e Log-Pearson Tipo III, para o munícipio de Francisco Beltrão, no Paraná.

\section{MATERIAL E MÉTODOS}

Os dados utilizados no presente estudo foram obtidos dos registros pluviométricos da Estação Francisco Beltrão, do Instituto Agronômico do Paraná - IAPAR, em Francisco Beltrão, Paraná. A estação climatológica está localizada nas coordenadas geográficas de $26^{\circ}$ 04'59" de latitude Sul, 53 03'00" de longitude Oeste e $650 \mathrm{~m}$ de altitude (Figura 1). O clima de Francisco Beltrão é classificado como STPU (CAMARGO, 1991). 


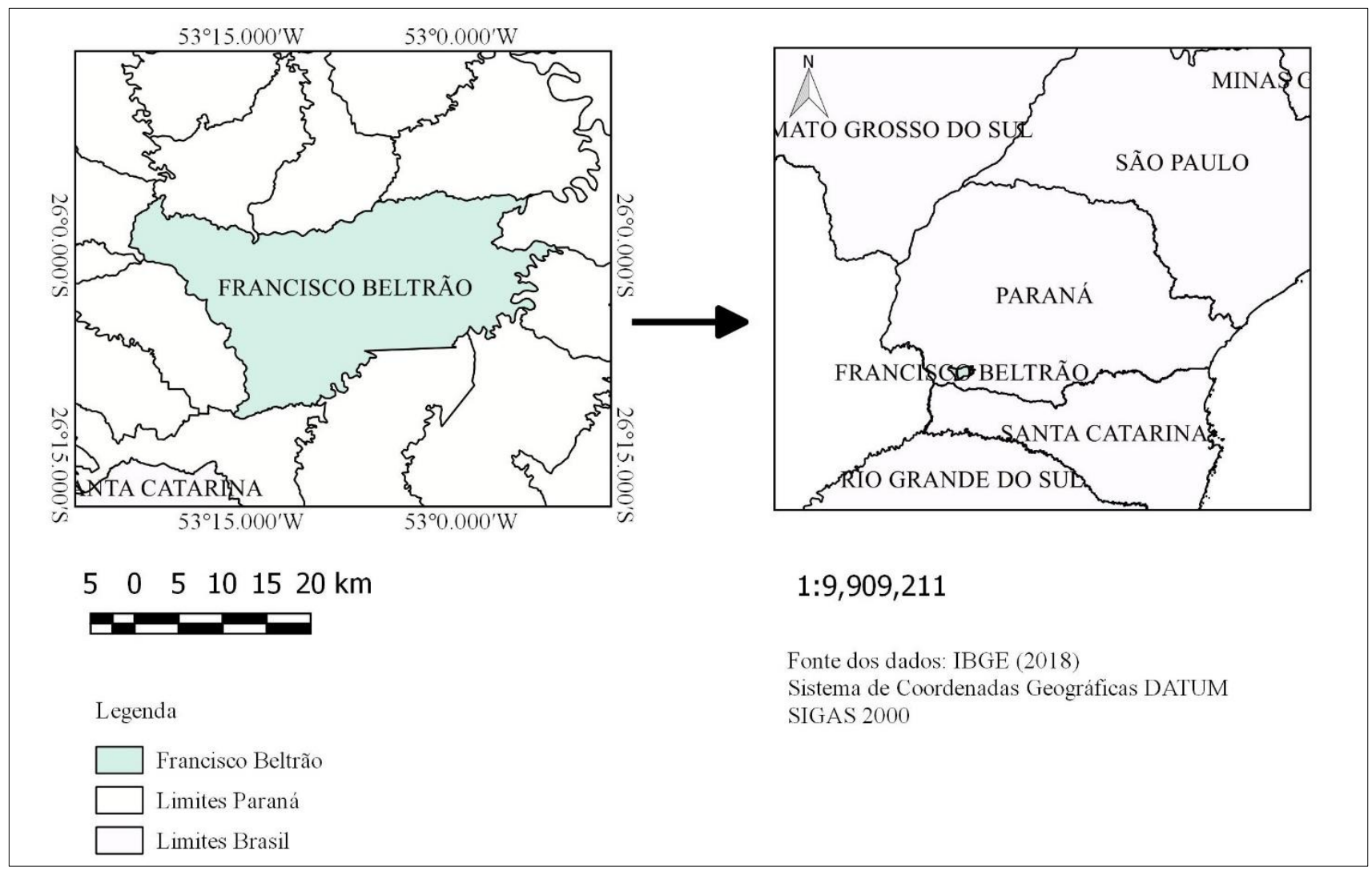

Figura 1. Mapa de localização da área de estudo, Francisco Beltrão-PR.

Foram utilizados dados de precipitação pluviométrica máxima anual de 1974 a 2017 (44 anos), os dados foram organizados anualmente e posteriormente foram feitos os cálculos do período de retorno pelas distribuições de Gumbel e Log Pearson III, através das séries de precipitações pluviométricas máximas utilizadas no período avaliado. Foram utilizados os períodos de retorno de 2, 5, 10, 20, 50 e 100 anos.

A distribuição de probabilidade de Gumbel é aplicada às séries históricas de valores extremos, especialmente, a precipitação máxima diária. Sua Função Cumulativa de Probabilidades (FCP) é representada pela Equação 1 (BERTONI; TUCCI, 2015).

$$
\mathrm{FCP}: \mathrm{P}(\mathrm{X} \leq \mathrm{x})=\exp \left(-\exp \left(-\left(\frac{-\mathrm{x}-\alpha}{\beta}\right)\right)\right)
$$

Em que:

FCP: Função Cumulativa de Probabilidades;

$\alpha$ : Parâmetro de escala;

$\beta$ : Parâmetro de forma.

$\mathrm{X}$ : Valores de precipitação máxima diária anual.
Os parâmetros são encontrados pelas equações 2 e 3 .

$$
\begin{aligned}
& \beta=\left(\frac{\sqrt{6}}{\pi}\right) . S \\
& \alpha=\bar{x}-0,5772 \cdot \beta
\end{aligned}
$$

Em que:

$\mathrm{S}=$ Desvio Padrão das precipitações;

$\bar{x}=$ Média das precipitações.

O tempo de retorno é calculado pela equação 4.

$$
\mathrm{TR}=\frac{1}{1-\mathrm{p}}
$$

Em que:

TR = Tempo de retorno, em anos; $\mathrm{P}=$ Probabilidade do evento acontecer (adimensional).

A equação 5 representa a estimativa da representação máxima em função dos parâmetros $\alpha$ e $\beta$, além do período de retorno. 
$X=\beta-\operatorname{Ln}\left(-\operatorname{Ln}\left(1-\frac{1}{\mathrm{TR}}\right)\right) \cdot \alpha$

A função densidade de probabilidade que representa uma distribuição Pearson Tipo III é dada pela equação 6 (MELLO; SILVA, 2013).

$f(x)=\frac{1}{\alpha x \Gamma(\beta)}\left\{\ln \frac{x-y}{\alpha}\right\}^{\beta-1} e^{-\left\{\frac{\ln x-y}{\alpha}\right\}} x \geq \gamma$

Em que:

$\alpha e \beta=$ Parâmetros da distribuição;

$\Gamma=$ Função gama.

Os dados foram submetidos à análise estatística com o auxílio do software R 2.15 e os gráficos gerados pelo software Minitab 17.

\section{RESULTADOS E DISCUSSÃO}

A Figura 2 demonstra a precipitação máxima em função do tempo de retorno pela distribuição de Gumbel.

Pode-se comportamento retorno, com uma correlação quadrática forte $\left(r^{2}=92,7\right)$.

Sansigolo (2008) ao estudar diversas distribuições teóricas de probabilidade, concluiu que a distribuição de Gumbel melhor se ajustou as precipitações máximas de Piracicaba, São Paulo.
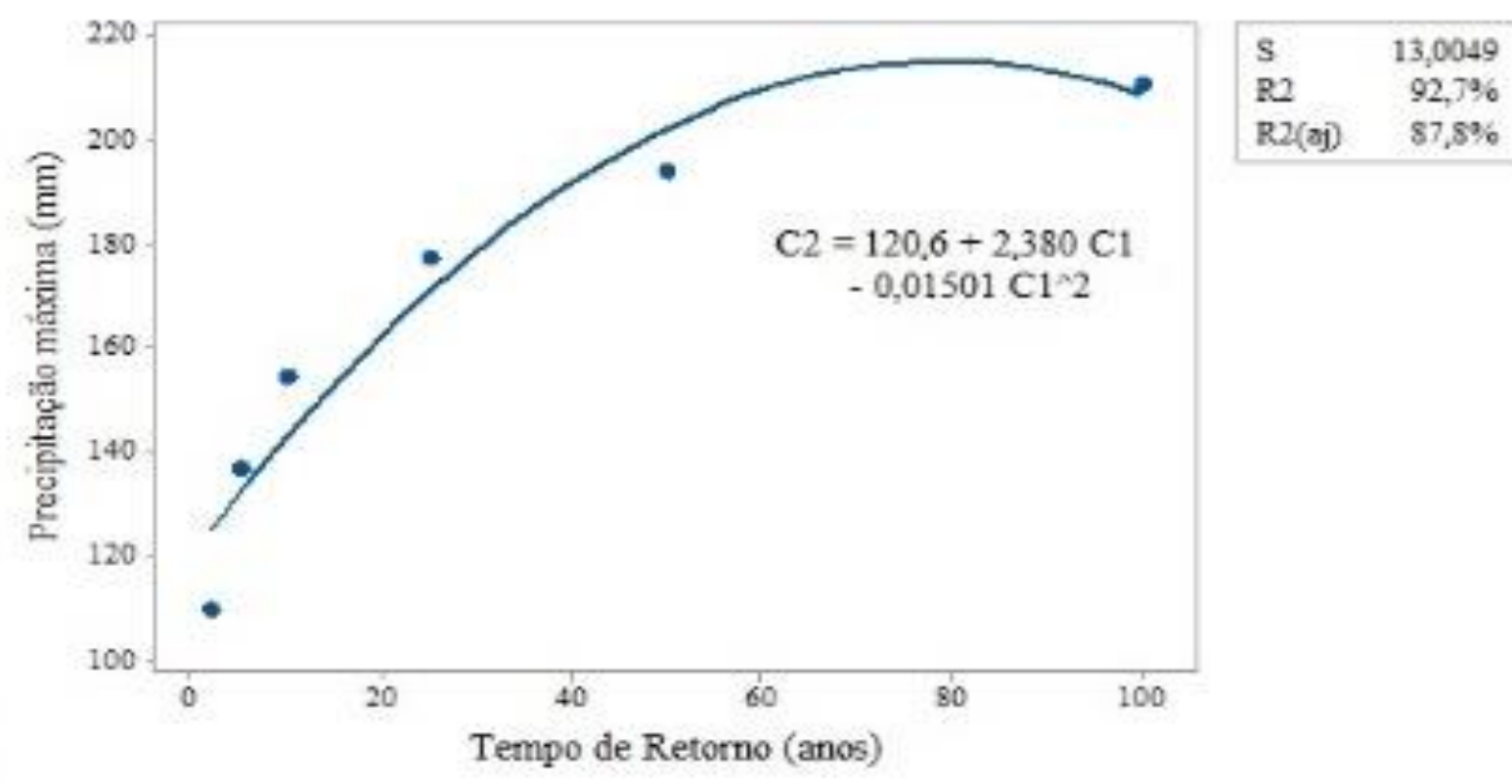

Figura 2. Precipitação máxima em função do tempo de retorno pela distribuição de Gumbel.

O mesmo comportamento foi observado por Freire et al. (2013), ao estudarem precipitações máximas prováveis em diferentes tipos de retorno utilizando a distribuição de Gumbel para o município de São João do Jaguaribe no Ceará. Tais resultados corroboram com Guimarães et al. (2015), que ao estudar precipitações máximas para o município de Cruz das Almas, BA para os tempos de retorno de $2,5,10,20,30,50,100,200$ e 300 anos com o modelo probabilístico de Gumbel, concluiu que os dados geraram um modelo probabilístico satisfatório.

Na Figura 3, verifica-se o comportamento das precipitações máximas em função do período de retorno pela distribuição de Log Pearson III. Assim como ocorreu na distribuição Gumbel, a precipitação variou de acordo com o período de retorno e houve uma correlação quadrática forte $\left(r^{2}=91,7\right)$. 


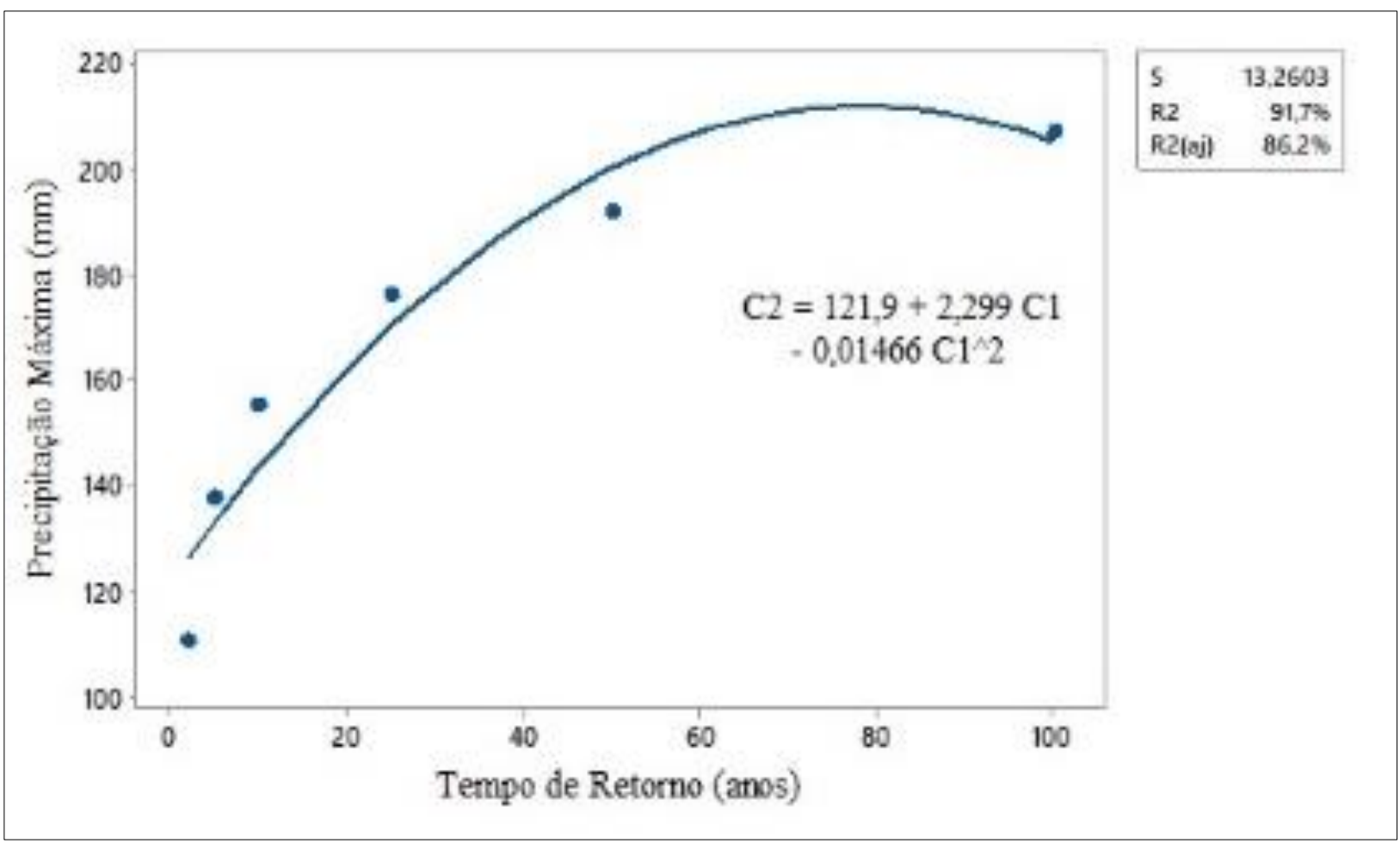

Figura 3. Precipitação máxima em função do tempo de retorno pela distribuição de Log Pearson III.

De acordo com os resultados obtidos, observa-se que o comportamento da precipitação máxima em função do período de retorno varia com o passar dos anos. Para os menores períodos de retorno estudados $(2,5 \mathrm{e}$ 10 anos), a Distribuição de Log Pearson III apresentou maiores valores de precipitação máxima quando comparado a Distribuição de Gumbel, O mesmo comportamento foi observado por Martins et al. (2011) para o município de Anchieta-ES, que para menor tempo de retorno ( 2 anos) obteve uma maior precipitação máxima com a distribuição de Log Pearson III, e com tempos de retorno maiores (50 e 100) obteve uma maior precipitação máxima com a distribuição Gumbel.

\section{CONCLUSÕES}

Em ambas distribuições de probabilidade, as precipitações variaram de acordo com o período de retorno.

Para os períodos de retorno de 2, 5 e 10 anos, a distribuição de Log Pearson III, apresentou maiores valores de precipitação máxima e para os períodos de retorno de 25,50 e 100 anos, a distribuição de Gumbel apresentou maiores valores de precipitação máxima.

As distribuições de Gumbel e Log Pearson III apresentaram comportamento semelhante, com uma correlação forte, porém a distribuição de Gumbel obteve um melhor ajuste dos dados $\left(r^{2}=92,7 \%\right)$.

\section{REFERÊNCIAS BIBLIOGRÁFICAS}

ALVES, A. V. P.; SANTOS, G. B. S.; MENEZES FILHO, F. C. M.; SANCHES, L. Análise dos métodos de estimativa para os parâmetros das distribuições de Gumbel e GEV em eventos de precipitações máximas na cidade de Cuiabá-MT. Revista Eletrônica de Engenharia Civil, v. 6, n. 1, p. 32-43, 2013. https://doi.org/10.5216/reec.v6il.21635.

BACK, A. J. Seleção de distribuição de probabilidade para chuvas diárias extremas do 
estado de Santa Catarina. Revista Brasileira de Meteorologia, v. 16, n. 2, p. 211-222, 2001.

BARRETO, H. B. F.; PEREIRA, G. M.; BARRETO, F. P.; FREIRE, F. G. C.; MAIA, P. M. E. Relação intensidade duração frequência para precipitação extrema em Mossoró-RN. Global Science and Technology, v. 7, n. 3, p. 103-109, 2014. https://doi.org/10.14688/19843801/GST.V7N3P103-109.

BERTONI, J. C.; TUCCI, C. E. M. Precipitação. In: TUCCI, C. E. M. (Org.). Hidrologia: ciência e aplicação. 4. ed. Porto Alegre: ABRH ; UFRS, 2015. p.177-241.

BESKOW, S.; CALDEIRA, T. L.; MELLO, C. R.; FARIA, L. C.; GUEDES, H. A. S. Multiparameter probability distributions for heavy rainfall modeling in extreme Southern Brazil. Journal of Hidrology: Regional Studies, v. 4, p. 123-133, 2015. https://doi.org/10.1016/j.ejrh.2015.06.007.

CAMARGO, A. P. de. Classificação climática para zoneamento de aptidão agroclimática. Revista Brasileira de Agrometeorologia, v. 8, p. 126-131, 1991.

CARVALHO, A. L.; SOUZA, J. L.; LYRA, G. B.; PORFIRIO, A. C. S. FERREIRA JUNIOR, R. A. Ocorrência de períodos secos na região de Rio Largo. Revista Brasileira de Meteorologia, v. 28, n. 2, p. 173-180, 2013. http://dx.doi.org/10.1590/S0102-

77862013000200006 .

COTTA, H. H. A.; CORREA, W. S. C.; ALBUQUERQUE, T. T. A. Aplicação da distribuição de Gumbel para valores extremos de precipitação no município de Vitória, ES. Revista Brasileira de Climatologia, v. 19, p. 203-217,2016. http://dx.doi.org/10.5380/ abclima.v19i0.39440.

FREIRE, F. G. C.; BARRETO, H. B. F.; BATISTA, R. O.; SANTOS, W. O. Estudo das precipitações para diferentes períodos de retorno no município de São João do Jaguaribe. Revista Brasileira de Agricultura Irrigada, v. 7, n. 5, p. 300-305, 2013. http://dx.doi.org/10.7127/RBAI.V7N500169.

GUIMARÃES, M. J. M.; LOPES, I.; SILVA, W. P.; SILVA, A. S. Estudo das precipitações Máximas para o município de Cruz das Almas, BA. Magistra, v. 27, n. 3, p. 326-332, 2015.

MARTINS, C. A. S.; REIS, E. M. U.; REIS, E. F. Estimativa da vazão e da precipitação máxima utilizando modelos probabilísticos na bacia hidrográfica do rio Benevente. Enciclopédia Biosfera, v. 7, n. 13, p. 11301142, 2011.

MELlO, C. R.; SILVA, A. M. Hidrologia: Princípios e aplicações em sistemas agrícolas. 1 ed. Lavras: Editora UFLA, 2013. $455 \mathrm{p}$.

MIRANDA, C. T. S.; THEBALDI, M. S.; ROCHA, G. M. R. B. Precipitação máxima diária anual e estimativa da equação de chuvas intensas do município de Divinópolis, MG, Brasil. Scientia Agraria, v. 18, n. 4, p. 09-16, 2017.

http://dx.doi.org/10.5380/rsa.v18i4.49883.

MORUZZI, R. B.; OLIVEIRA, R. B. Relação entre intensidade, duração e frequência de chuvas em Rio Claro, SP: métodos e aplicação. Teoria e Prática na Engenharia Civil, n. 13, p. 59-68, 2009.

SANSIGOLO, C. A. Distribuições de extremos de precipitação diária, temperatura máxima e mínima e velocidade do vento em Piracicaba, SP. Revista Brasileira de Meteorologia, v. 23, n. $\quad 3, \quad$ p. $341-346, \quad 2008$. http://dx.doi.org/10.1590/S010277862008000300009 .

VALVERDE, A . E. L.; LEITE, H. G.; SILVA, D. D.; PRUSKI , F. F. Momentos-L: Teoria e aplicação em hidrologia. Revista Árvore, v. 2 8, n. 6, p. 927 933, 2004. http://dx.doi.org/ 10.1590/ S0100-67622004000600019. 